oncological outcomes particularly in renal cancer and renal surgery. Moreover, the complication rates with the laparoscopic technique have been demonstrated to be comparable to the open technique.

\author{
Dr. Fernando J. Kim \\ Chief of Urology, Denver Health Med. Ctr. \\ Associate Professor, Univ. Colorado Health Sci. Ctr. \\ Director of Minimally Invasive Urol. Oncology, UCHSC \\ Denver, Colorado, USA \\ E-mail:fernando.kim@dhha.org
}

doi: $10.1590 / S 1677-553820100001000017$

\title{
Cost analysis of robotic versus open radical cystectomy for bladder cancer
}

Smith A, Kurpad R, Lal A, Nielsen M, Wallen EM, Pruthi RS

Division of Urologic Surgery, The University of North Carolina at Chapel Hill, Chapel Hill, North Carolina, USA

J Urol. 2010; 183: 505-9

Purpose: Recently robotic approaches to cystectomy have been reported, and while clinical and oncological efficacy continues to be evaluated, potential financial costs have not been clearly evaluated. In this study we present a financial analysis using current cost structures and clinical outcomes for robotic and open cystectomy for bladder cancer.

Materials and Methods: The financial costs of robotic and open radical cystectomy were categorized into operating room and hospital components, and further divided into fixed and variable costs for each. Fixed operating room costs for open cases involved base cost as well as disposable equipment costs while robotic fixed costs included the amortized machine cost as well as equipment and maintenance. Variable operating room costs were directly related to length of surgery. Variable hospital costs were directly related to transfusion requirement and length of stay. The means of the prior 20 cases of robotic and open cystectomy were used to perform a comparative cost analysis.

Results: Mean fixed operating room costs for robotic cases were \$1,634 higher than for open cases. Operating room variable costs were also higher by a difference of $\$ 570$, directly related to increased operating room time. Hospital costs were nearly identical for the fixed component while variable costs were $\$ 564$ higher for the open approach secondary to higher transfusion costs and longer mean length of stay. Based on these findings robotic cystectomy is associated with an overall higher financial cost of $\$ 1,640$ (robotic $\$ 16,248$ vs open $\$ 14,608)$. Cost calculators were constructed based on these fixed and variable costs for each surgical approach to demonstrate the expected total costs based on varying operating room time and length of stay.

Conclusions: Robotic assisted laparoscopic radical cystectomy is associated with a higher financial cost $(+\$ 1,640)$ than the open approach in the perioperative setting. However, this analysis is limited by its single institution design and a multicenter followup study is required to provide a more comprehensive analysis.

\section{Editorial Comment}

Independently of the techniques used for the surgical treatment of bladder cancer, the oncological principles must be followed and outcomes ought to be equal or exceed the tumor control and improve the recovery time. 
This article demonstrates that robotic radical cystectomy has similar short-term cancer control and complication rates, less operative time and a shorter hospital stay than laparoscopic or open radical cystectomy. The authors recently performed completely intracorporeal robotic cystectomy and diversion setting the bar for minimally invasive radical cystectomy and urinary diversion very high. It will be important for other centers to duplicate these results and take into account the price of acquiring the robot and servicing it plus the cost of disposables utilized during robotic surgery.

Dr. Fernando J. Kim

Chief of Urology, Denver Health Med. Ctr. Associate Professor, Univ. Colorado Health Sci. Ctr. Director of Minimally Invasive Urol. Oncology, UCHSC

Denver, Colorado, USA

E-mail:fernando.kim@dhha.org 